\title{
Atom-Orbital Qubit under Non-Adiabatic Holonomic Quantum Control
}

\author{
Hongmian Shui ${ }^{\ddagger},{ }^{1}$ Shengjie Jin ${ }^{\ddagger},{ }^{1}$ Zhihan $\mathrm{Li}^{\ddagger},{ }^{1}$ Fansu Wei,${ }^{1}$ Xuzong Chen, ${ }^{1}$ Xiaopeng Li, ${ }^{2,}, 3, *$ and Xiaoji Zhou ${ }^{1,4, \dagger}$ \\ ${ }^{1}$ State Key Laboratory of Advanced Optical Communication System and Network, \\ Department of Electronics, Peking University, Beijing 100871, China \\ ${ }^{2}$ State Key Laboratory of Surface Physics, Institute of Nanoelectronics and Quantum Computing, \\ and Department of Physics, Fudan University, Shanghai 200438, China \\ ${ }^{3}$ Shanghai Qi Zhi Institute, Shanghai 200030, China \\ ${ }^{4}$ Collaborative Innovation Center of Extreme Optics, \\ Shanxi University, Taiyuan, Shanxi 030006, China
}

(Dated: December 10, 2021)

\begin{abstract}
Quantum computing has been attracting tremendous efforts in recent years. One prominent application is to perform programmable quantum simulations of electron correlations in large molecules and solid-state materials, where orbital degrees of freedom are crucial to quantitatively model electronic properties. Electron orbitals unlike quantum spins obey crystal symmetries, making the atomic orbital in optical lattices a natural candidate to emulate electron orbitals. Here, we construct an atom-orbital qubit by manipulating $s$ - and $d$-orbitals of atomic Bose-Einstein condensation in an optical lattice. Noise-resilient single-qubit gates are achieved by performing holonomic quantum control, which admits geometrical protection. We find it is critical to eliminate the orbital leakage error in the system. Our work opens up wide opportunities for atom-orbital based quantum information processing, of vital importance to programmable quantum simulations of multi-orbital physics in molecules and quantum materials.
\end{abstract}

Orbital degrees of freedom are essential to the quantitative description of electrons in large molecules [1] and solid state materials [2]. The complex interplay of spin, charge, and orbital, is key to the emergence of novel electron phenomena such as multiferroics [2], unconventional superconductivity [3], and exotic molecular spin filtering $[4,5]$. Incorporating orbitals lies at the heart of accurate quantum chemistry calculations [1], and adds substantial computation complexity in simulating manybody electron correlation. With quantum computing, the overall computation complexity of quantum algorithms for quantum chemistry calculation has been reduced to polynomial [6, 7], which has triggered much recent research effort on experimental demonstration of such quantum algorithms with superconducting qubits $[8,9]$ and trapped ions $[10,11]$. However, using these qubits to emulate electron orbitals meets experimental challenge of expensive qubit encoding [8] and demanding Hamiltonian engineering to impose precise orbital symmetry [12], and consequently the captured multi-orbital effect in the experiments is rather limited $[8,9]$.

With ultracold atoms confined in optical lattices, the atom-orbital wavefunction obeys the same crystalline symmetry as the electron orbitals [13], making atom-orbital an ideal qubit candidate to perform programmable quantum simulations of electron orbitals in molecules and solid materials. There has been fascinating progress accomplished in controlling atom orbitals in optical lattices for quantum simulations of exotic superfluids [14-19] and topological quantum states [20-23]. However, their gate-based universal quantum control is still lacking, leaving orbital qubit quantum computing so-far unachieved.

In this Letter, we construct an atom-orbital qubit us- ing $s$ - and $d$-orbitals of a one-dimensional optical lattice. We have measured the orbital relaxation time $\left(T_{\text {relax }}\right)$ and the dephasing time $\left(T_{2}\right)$, finding $T_{\text {relax }}=4.5 \pm 0.1$ ms (milliseconds), and $T_{2}=2.1 \pm 0.1 \mathrm{~ms}$ in our experiment. By programming lattice modulation, we reach universal nonadiabatic holonomic quantum gate control $[24,25]$ over the atom-orbital qubit, which exhibits noise-resilience against laser fluctuations due to geometrical protection. We demonstrate the holonomic quantum control of Hadamard and $\pi / 8$ gates, which form a universal gate-set for single-qubit rotation. The lattice modulation pulses are programmed to minimize orbital leakage error, which is the key to reach high fidelity holonomic quantum control of atom-orbital qubit. We implement quantum process tomography (QPT) on the orbital qubit to measure the full density matrix, from which the obtained average gate fidelity is $98.36(10) \%$.

Atom-orbital qubit. - Our experiment is based on a ${ }^{87} \mathrm{Rb}$ Bose-Einstein condensate (BEC) with $2 \times 10^{5}$ atoms confined in a one-dimensional (1D) optical lattice. The lattice potential takes a form of $V_{p}(x)=V_{0} \cos ^{2}(2 \pi x / l)$, with $V_{0}$ the lattice depth, and $l$ the wavelength of the laser forming the lattice, which is $1064 \mathrm{~nm}$ in this experiment. The lattice depth $V_{0}$ is five times of the laser recoil energy $\left(E_{r}=h^{2} / 2 m l^{2}\right)$, with $m$ the atomic mass. With lattice confinement, atoms acquire orbital degrees of freedom, as each lattice site contains localized Wannier orbitals $-s, p, d, \ldots$ [13], whose quantum superposition gives multiple Bloch bands. Having atoms condense at lattice quasi-momentum $k=0$, the orbital quantum state is described by a density matrix

$$
\rho=\sum_{\nu \nu^{\prime}} \rho_{\nu \nu^{\prime}}|\nu\rangle\left\langle\nu^{\prime}\right|,
$$

where $|\nu=s, p, d, \ldots\rangle$ represents the Bloch mode at 

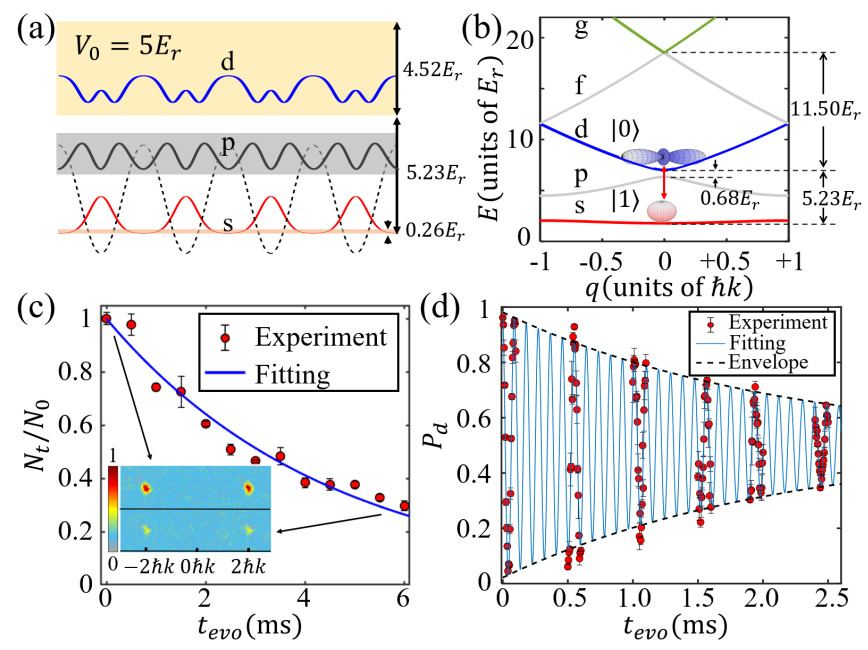

FIG. 1. Atom orbital qubit in a one-dimensional optical lattice. (a) The Bloch wave function amplitude of the $s, p$, and $d$ bands at zero quasi-momentum. The $s$ - and $d$ - states constitute our orbital qubit. The dashed line represents the optical lattice potential. (b) The band gap structures in our experimental optical lattice. The energy bands from bottom to top are $s, p, d, f$ and $g$ bands respectively. (c) Measurement of relaxation time, $T_{\text {relax }}$, in the decay dynamics of $d$-band population. The red dots correspond to the time evolution of the atom number $N_{d}$ in the $d$ band by timeof-flight measurement. The blue line represents fitting to a function $N_{d}(t)=N_{0} \exp \left(-t / T_{\text {relax }}\right)$. The relaxation time is determined as $T_{\text {relax }}=4.5 \mathrm{~ms}$. (d) Measurement of dephasing time, $T_{2}$, through Ramsey interference. The red dots show the measured time evolution of the proportion of atoms $P_{d}$ in the $d$-band by band mapping method. The blue line represents a fitting function $P_{d}=A \exp \left(-t / T_{2}\right) \sin (\omega t+\phi)+B$, with the black line the envelope of the Ramsey oscillations. We have a dephasing time $T_{2}=2.1 \mathrm{~ms}$ by fitting.

$k=0$ of the $\nu$-orbital band. In order to control the orbital state, we apply lattice modulation with a particular frequency $\omega$ to resonantly couple $s$ and $d$ orbitals (see Fig. 1), which introduces an additional potential,

$$
\Delta V(x, t)=A \sin (\omega t+\varphi) V_{p}(x),
$$

with both of amplitude $A$, and phase $\varphi$ programmable in our experiment (see Supplementary Material). With leakage to other orbitals neglected, the system corresponds to a two-level system, defining our atom-orbital qubit, with $|d\rangle$ and $|s\rangle$ orbital states identified as the qubit basis states, $|0\rangle$ and $|1\rangle$. In our experimental lattice setup, the energy gap between $s$ - and $d$-bands has a large detuning from the energy gap between other orbitals except the gap from the $s$ - to $p$-band. Despite the absence of the energy suppression of the $s$ to $p$ transition, this transition is forbidden due to inversion symmetry present during the lattice modulation. The transitions from the $s$-orbital to the undesired orbitals are thus avoided, either by energy suppression or by symmetry.

For qubit initialization, we selectively load the atomic
BEC into the lattice using our previously developed shortcut preparation method [26], with which we are able to initialize the qubit to an arbitrary state, $|\psi\rangle=$ $\cos \theta|0\rangle+\sin \theta e^{i \phi}|1\rangle$, within $250 \mu \mathrm{s}$. For qubit readout, we implement a time-of-flight quantum state tomography (TOFQST), which extracts the full information of the density matrix $\rho_{\nu \nu^{\prime}}$ from the atomic momentum distribution (see Supplemental Material). In the experiment, we prepare a set of six complementary initial states, $\mathbb{S} \equiv\left\{|0\rangle,|1\rangle,|+\rangle=\frac{1}{\sqrt{2}}(|0\rangle+|1\rangle),|-\rangle=\right.$ $\left.\frac{1}{\sqrt{2}}(|0\rangle-|1\rangle),|+i\rangle=\frac{1}{\sqrt{2}}(|0\rangle+i|1\rangle),|-i\rangle=\frac{1}{\sqrt{2}}(|0\rangle-i|1\rangle)\right\}$, whose measured state fidelities are $99.96(2) \%, 99.88(5) \%$, 99.30(11), 99.37(10)\%, 99.83(5)\%, and 99.98(1)\%. The averaged fidelity is $99.72(7) \%$.

By initializing to the excited $d$-orbital state, namely $|0\rangle$, we measure the time $(t)$ evolution of the atom number $N_{d}$ in the $d$ band, and obtain $N_{d}(t)$. Fitting this function to $e^{-t / T_{\text {relax }}}$, we extract the relaxation $T_{\text {relax }}$ time, which is $4.5 \pm 0.1 \mathrm{~ms}$ (Fig. 1(c)). To measure the dephasing time $T_{2}$, we prepare an orbital superposition state $[|0\rangle+|1\rangle] / \sqrt{2}$. For the energy splitting between $s$, and $d$ orbital bands in the lattice, this superposition state develops Ramsey interference fringes in dynamics. The time dependence of the off-diagonal term has a form of (Fig. 1(d))

$$
\operatorname{Re}\left(\rho_{s d}(t)\right) \sim e^{-t / T_{2}} \cos (\Omega t+\phi),
$$

with $\Omega, \phi, T_{2}$ determined by fitting the experimental data gotten by band mapping method. We obtain a $T_{2}$ time of $2.1 \pm 0.1 \mathrm{~ms}$.

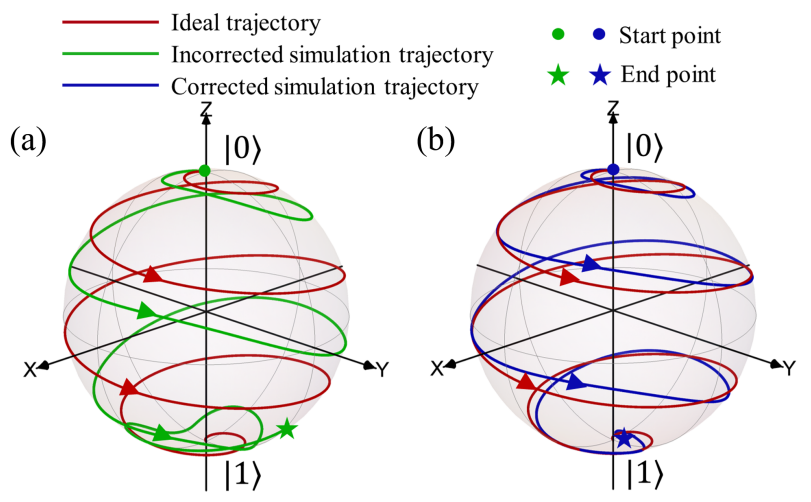

FIG. 2. Simulated time evolution of the $|0\rangle$ state on the Bloch sphere under the holonomic $X$ gate. North and south poles of Bloch sphere denote the $|d\rangle$ and $|s\rangle$ orbital states. The red curve shows the ideal state trajectory under the holonomic control according to the idealized qubit model (Eq. (4)). The blue (green) curve shows the state trajectory by the multiorbital numerical simulation, with (without) the orbital leakage error eliminated.

Nonadiabatic holonomic orbital gate construction.With the optical potential in Eq. (2), we have a qubit 
control Hamiltonian $H(t)$ of the form,

$$
\frac{1}{2} \Delta \sigma_{z}+\frac{1}{2} \lambda\left(-\cos (\omega t+\varphi) \sigma_{y}+\sin (\omega t+\varphi) \sigma_{x}\right),
$$

where $\Delta$ is the gap between the $s$ and $d$-bands at quasimomentum $k=0\left(\Delta=5.23 E_{r}\right.$ in our experiment), and the induced coupling by the lattice modulation is

$$
\lambda=A \int d x V_{p}(x) \phi_{d}(x) \phi_{s}(x),
$$

with $\phi_{\nu=s, d}(x)$ the Bloch function of $\nu$-orbital band at $k=0$. With a resonant coupling $\omega \approx \Delta$, the overall quantum gate operation time is determined by $1 / \lambda$. To avoid non-resonant transitions to other bands, predominately the $g$-band, which has an energy gap from the $s$-band, $\Delta_{s g}\left(=16.73 E_{r} \gg \Delta\right)$, it is required that $\lambda \ll\left|\Delta_{s g}-\omega\right|$, which sets an upper limit for the amplitude of lattice modulation. For this requirement, it becomes more desirable to construct nonadiabatic quantum gates, as the adiabatic control would be too slow-the total adiabatic evolution time is required to be much longer than $1 / \lambda$ to maintain quantum adiabaticity.

We implement nonadiabatic holonomic orbital control based on a dynamical invariant of the Hamiltonian in Eq. (4), $I=(\Delta-\omega) \sigma_{z}+$ $\lambda\left(-\cos (\omega t+\varphi) \sigma_{y}+\sin (\omega t+\varphi) \sigma_{x}\right) \quad[24, \quad 25], \quad$ with its instantaneous eigenstates $\left|\psi_{ \pm}(t)\right\rangle$. Through one period $(T=2 \pi / \omega)$ of quantum evolution, an initial quantum state $\sum_{n} c_{n}\left|\psi_{n}\right\rangle$ is transformed to $\sum_{n} c_{n} e^{i\left(\gamma_{n}^{g}+\gamma_{n}^{d}\right)}\left|\psi_{n}\right\rangle$, with the accumulated geometrical phase $\gamma_{n}^{g}=\int_{0}^{T} d t\left\langle\psi_{n}(t)\left|i \partial_{t}\right| \psi_{n}(t)\right\rangle$, and the dynamical phase $\gamma_{n}^{d}=-\int_{0}^{T} d t\left\langle\psi_{n}(t)|H(t)| \psi_{n}(t)\right\rangle$. The wavefunction evolution in the dynamical invariant eigenbasis during the holonomic quantum control resembles quantum adiabatic dynamics $[24,25]$. The geometrical phase only depends on the solid angle enclosed by the evolution path of $\left|\psi_{n}(t)\right\rangle$ on the Bloch sphere [27]. The holonomic gate is thus a generalization of adiabatic geometrical gate [28]. This gate has intrinsic resilience against experimental control errors for geometrical protection [29, 30], which has been demonstrated with liquid NMR [31,32], solid-state [33-38], neutral atoms [39-41], ions [42-46] and superconducting qubits [47-52]. To exploit the geometrical protection, the dynamical phase has to be cancelled, which corresponds to

$$
\lambda^{2}+\Delta(\Delta-\omega)=0 .
$$

This gives the holonomic gate condition [25], which is explicitly satisfied in our lattice modulation design. The $\mathrm{SU}(2)$ rotation through one period of lattice modulation is then $U_{\beta \varphi}(T)=\sum_{ \pm} e^{i \gamma_{ \pm}^{g}}\left|\psi_{ \pm}(0)\right\rangle\left\langle\psi_{ \pm}(0)\right|$. This singlequbit rotation can be rewritten as

$$
U_{\beta \varphi}=-e^{i \pi \sin \beta\left[-\sin \varphi \cos \beta \sigma_{x}+\cos \varphi \cos \beta \sigma_{y}+\sin \beta \sigma_{z}\right]}
$$

with $\beta$ determined by the lattice modulation frequency$\cos ^{2} \beta=\Delta / \omega(\beta \in[0, \pi / 2])$, and the angle $\varphi$ controllable by programming the potential (Eq. (2)). An arbitrary target of holonomic $\mathrm{SU}(2)$ rotation, $U_{\text {target }}$, is approached by combining multiple periods ( $M$ times) of lattice modulation, which gives a concatenated unitary $U=U_{\beta_{M} \varphi_{M}} U_{\beta_{M-1} \varphi_{M-1}} \ldots U_{\beta_{2} \varphi_{2}} U_{\beta_{1} \varphi_{1}}$. The parameter sequence $\left(\beta_{j}, \varphi_{j}\right)$ is determined by optimizing the gate fidelity $F=\left|\operatorname{tr}\left(U^{\dagger} U_{\text {target }}\right)\right| / 2$. We then obtain a control sequence of lattice modulation frequency, amplitude, and phase, denoted by $\Theta_{j} \equiv\left(\omega_{j}, A_{j}, \varphi_{j}\right)$. With this scheme, we expect gate fidelities above $99.999 \%$ using $M \leq 5$ for $X, Y, Z$, Hadamard, and $\pi / 8$ gates under the idealized model in Eq. (4)).
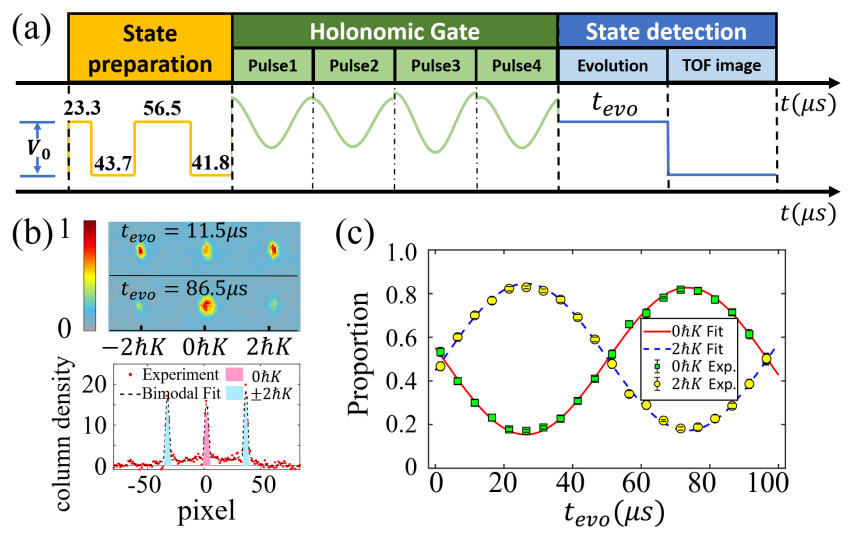

FIG. 3. Experimental realization of the holonomic quantum gates. (a) Experimental procedures of performing holonomic $X$ gate on the initial state, $|-i\rangle$. The numbers on top of the square pulses in (a) represent the time durations in units of microseconds. (b) Time-of-flight patterns at different evolution time. The condensed numbers of atoms at momentum $0 \hbar K$, and $\pm 2 \hbar K$ are extracted following standard analysis in cold atom experiments. (c) Oscillation dynamics of the atom number proportion at $0 \hbar K$ and $\pm 2 \hbar K$. We average over four experimental runs for each data point, with the error bar denoting the standard statistical error. Here we set the lattice confinement $V_{0}=5 E_{r}$.

However, in comparing the results of the ideal model with a more precise multi-orbital numerical simulation that incorporates all continuous degrees of freedom of the lattice (Supplementary Material), we find significant discrepancy. Fig. 2 illustrates one example of state evolution under a constructed holonomic $X$ gate. In the example shown in Fig. 2(a), the gate fidelity obtained by the multi-orbital numerical simulation is below $85 \%$. The sizeable difference from the ideal model is attributed to the leakage to other unwanted orbitals. Even a small fraction of $g$-band population below $5 \%$ is found to strongly disturb the qubit evolution on the Bloch sphere (Fig. 2(a)). To resolve the problem of orbital leakage, we develop an orbital leakage elimination protocol (Supplemental Material), where the leakage error is minimized by optimizing the control sequence $\Theta_{j}$. 


\begin{tabular}{cccccccc}
\hline Initial state & $|0\rangle$ & $|1\rangle$ & $|+\rangle$ & $|-\rangle$ & $|+i\rangle$ & $|-i\rangle$ & Average \\
\hline X-gate & $99.92(3)$ & $99.08(8)$ & $98.87(11)$ & $93.28(34)$ & $98.75(9)$ & $99.13(7)$ & $98.17(16)$ \\
Y-gate & $99.19(13)$ & $99.71(6)$ & $98.29(13)$ & $96.91(20)$ & $98.73(11)$ & $99.15(8)$ & $98.66(13)$ \\
Z-gate & $97.87(14)$ & $99.97(3)$ & $97.47(18)$ & $94.83(29)$ & $99.14(11)$ & $97.75(16)$ & $97.84(17)$ \\
Hadamard & $98.24(18)$ & $98.38(11)$ & $99.45(7)$ & $97.90(20)$ & $99.08(8)$ & $96.77(23)$ & $98.30(16)$ \\
$\pi / 8$-gate & $99.21(21)$ & $99.76(7)$ & $97.78(30)$ & $95.94(41)$ & $99.69(8)$ & $99.28(24)$ & $98.61(25)$ \\
\hline
\end{tabular}

TABLE I. Measured fidelities of the experimental holonomic quantum gates by TOFQST.

With the optimal control sequence designed for holonomic $X, Y, Z$, Hadamard, and $\pi / 8$ gates (Supplemental Material), the gate fidelity is improved to above $98 \%$ in the multi-orbital numerical simulation. The orbital leakage elimination protocol is implemented in our experiment for high fidelity realization of holonomic gates.

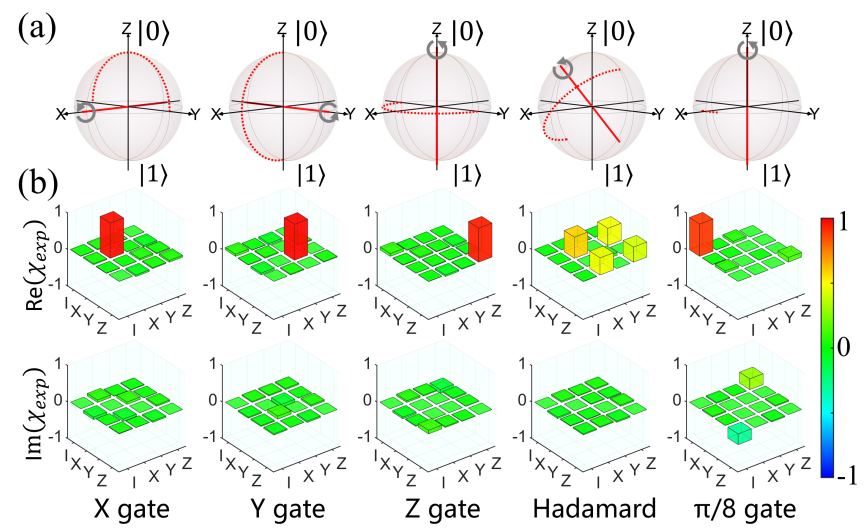

FIG. 4. Representations of the experimentally accomplished quantum processes. (a) The schematic illustration of the quantum processes. The red lines, gray arrows, and red dots, represent the rotation axis, direction and angle of the corresponding $\mathrm{SU}(2)$ rotation, respectively. (b) Process matrices of the implemented holonomic $X, Y, Z$, Hadamard, and $\pi / 8$ gates by QPT measurements.

Holonomic quantum orbital gate fidelities. - In the experiment, we implement holonomic orbital gates taking the control sequences as designed with our orbital leakage elimination protocol. The band population dynamics during the gate operation has a very good agreement with our multi-orbital numerical simulation (Supplementary Figure S5). Fig. 3 shows the experimental realization of the holonomic $X$ gate. The atomic BEC is initialized in the quantum state $|-i\rangle$. We then perform the holonomic $X$ gate control. The occupation of momentum $\tilde{p}=0$ and $\tilde{p}=2 \hbar K$ (with $K=2 \pi / l$ ) states are measured through time-of-flight (TOF), by which the full orbital qubit state is mapped out through TOFQST (Supplemental Material). This experimental procedure is repeated for all the initial states in $\mathbb{S}$. The fidelity $(F)$ averaged over the final states is $98.17(16) \%$ (Table I), as calculated from the overlap of density matrix measured in the experiment $\left(\rho_{\exp }\right)$ with the theoretical construction $\left(\rho_{\text {th }}\right)$, i.e., $F=\left|\operatorname{Tr}\left(\rho_{\exp } \rho_{\text {th }}^{\dagger}\right)\right| / \sqrt{\operatorname{Tr}\left(\rho_{\exp } \rho_{\exp }^{\dagger}\right) \operatorname{Tr}\left(\rho_{\text {th }} \rho_{\text {th }}^{\dagger}\right)}$.
We also implement the holonomic $Y, Z$, Hadamard, and $\pi / 8$ gates in the experiment, whose averaged final state fidelities are 98.66(13)\%, 97.84(17)\%, 98.30(16)\%, and 98.61(25)\%.

One way to construct universal holonomic gate for single qubit rotation is to combine Hadamard and $\pi / 8$ gates [53]. The other is to search for the control sequence numerically, whose universality is confirmed through our multi-orbital numerical simulation. We randomly sample a hundred Haar random $\mathrm{SU}(2)$ rotations, and find all can be constructed by the concatenated unitary sequence with $M \leq 5$, producing final state fidelities all above $98 \%$ (Supplementary Figure S2).

To characterize the holonomic quantum gates directly, we further perform quantum process tomography (QPT). We initialize the atomic BEC in the six orbital states in $\mathbb{S}$, and map out the final quantum states following the holonomic quantum gates by TOFQST. The $\chi$ matrices, which represent the quantum gate operations, are reconstructed by the QPT method $[54,55]$. The results are shown in Fig. 4. For completeness we also provide quantum process fidelities (QPFs) as defined by $\left|\operatorname{Tr}\left(\chi_{\exp } \chi_{\text {th }}^{\dagger}\right)\right| / \sqrt{\operatorname{Tr}\left(\chi_{\exp } \chi_{\text {exp }}^{\dagger}\right) \operatorname{Tr}\left(\chi_{\mathrm{th}} \chi_{\mathrm{th}}^{\dagger}\right)}$, with $\chi_{\exp }$ and $\chi_{\mathrm{th}}$, the $\chi$ matrix reconstructed from experimental measurements and the theoretical expectation, respectively. The measured QPFs are 98.47(9)\%, 98.35(11)\%, $97.81(13) \%, 98.53(8) \%, 98.63(15) \%$, for the X, Y, Z, Hadamard, and $\pi / 8$ gates in the experiment.

Discussion. - We have constructed an orbital qubit using atomic Bose-Einstein condensation in a onedimensional optical lattice. The atom orbital qubit is equipped with nonadiabatic holonomic quantum control, that shows noise resilience against laser intensity fluctuations due to geometrical protection (Supplementary Figure S3). In order to further improve the gate fidelity in the experiment, we expect it is helpful to incorporate the inhomogenity produced by the trap, which can be treated by integrating spin echo like pulse or optimal control protocols [56-58].

To achieve universal quantum computing with the orbit qubit setup, we still need to construct two-qubit gates, which can be implemented through an atom collision scheme (Supplementary Material). We expect the orbital quantum control techniques developed here can be generalized to full quantum control of all orbitals. For orbital transitions that respect parity symmetry, one 
may still take the lattice modulation protocol but using different resonant frequencies. To control orbital transitions violating parity symmetry would require lattice shaking techniques [59]. The orbital lattice based quantum computing would provide novel opportunities for programmable quantum simulations. For example, the demonstrated arbitrary coupling between $s$ and $d$ orbitals may be used to perform programmable quantum simulations of spin-orbital interaction analogue and the consequent topological physics [21].

Acknowledgements. - We acknowledge helpful discussion with Yidun Wan. This work is supported by National Natural Science Foundation of China (Grants No. 61727819, 11934002, 91736208, and 11774067), National Program on Key Basic Research Project of China (Grant No. 2016YFA0301501, Grant No. 2017YFA0304204), Shanghai Municipal Science and Technology Major Project (Grant No. 2019SHZDZX01).

$\ddagger$ These authors contributed equally to this work.

* xiaopeng_li@fudan.edu.cn

†xjzhou@pku.edu.cn

[1] R. Bartlett and M. Musiał, Rev. Mod. Phys. 79, 291 (2007).

[2] H. Hwang, Y. Iwasa, M. Kawasaki, B. Keimer, N. Nagaosa, and Y. Tokura, Nat. Mater. 11, 103 (2012).

[3] G. R. Stewart, Rev. Mod. Phys. 83, 1589 (2011).

[4] X. Li, J. Nan, and X. Pan, Phys. Rev. Lett. 125, 263002 (2020).

[5] Y. Liu, J. Xiao, J. Koo, and B. Yan, Nat. Mater. 20, 638 (2021).

[6] A. Aspuru-Guzik, A. Dutoi, P. Love, and M. HeadGordon, Science 309, 1704 (2005).

[7] S. McArdle, S. Endo, A. Aspuru-Guzik, S. Benjamin, and X. Yuan, Rev. Mod. Phys. 92, 015003 (2020).

[8] A. Kandala, A. Mezzacapo, K. Temme, M. Takita, M. Brink, J. Chow, and J. Gambetta, Nature (London) 549, 242 (2017).

[9] G. A. Quantum et al., Science 369, 1084 (2020).

[10] C. Hempel, C. Maier, J. Romero, J. McClean, T. Monz, H. Shen, P. Jurcevic, B. Lanyon, P. Love, R. Babbush, A. Aspuru-Guzik, R. Blatt, and C. Roos, Phys. Rev. X 8, 031022 (2018).

[11] Y. Nam, J.-S. Chen, N. Pisenti, K. Wright, C. Delaney, D. Maslov, K. Brown, S. Allen, J. Amini, J. Apisdorf, et al., npj Quantum Inform. 6, 1 (2020).

[12] J. McClean, J. Romero, R. Babbush, and A. AspuruGuzik, New J. Phys. 18, 023023 (2016).

[13] X. Li and W. Liu, Rep. Prog. Phys. 79, 116401 (2016).

[14] A. Isacsson and S. Girvin, Phys. Rev. A 72, 053604 (2005).

[15] W. Liu and C. Wu, Phys. Rev. A 74, 013607 (2006).

[16] A. B. Kuklov, Phys. Rev. Lett. 97, 110405 (2006).

[17] G. Wirth, M. Ölschläger, and A. Hemmerich, Nat. Phys. 7, 147 (2011).

[18] P. Soltan-Panahi, D.-S. Lühmann, J. Struck, P. Windpassinger, and K. Sengstock, Nat. Phys. 8, 71 (2012).

[19] S. Jin, W. Zhang, X. Guo, X. Chen, X. Zhou, and X. Li,
Phys. Rev. Lett. 126, 035301 (2021).

[20] K. Sun, W. Liu, A. Hemmerich, and S. Das Sarma, Nat. Phys. 8, 67 (2012).

[21] X. Li, E. Zhao, and W. Vincent Liu, Nat. Commun. 4, 1523 (2013).

[22] B. Liu, X. Li, B. Wu, and W. Liu, Nat. Commun. 5, 1 (2014)

[23] M. Khamehchi, C. Qu, M. Mossman, C. Zhang, and P. Engels, Nat. Commun. 7, 10867 (2016).

[24] H. Lewis and W. Riesenfeld, J. Math. Phys. 10, 1458 (1969).

[25] U. Güngördü, Y. Wan, and M. Nakahara, J. Phys. Soc. Jpn. 83, 034001 (2014).

[26] X. Zhou, S. Jin, and J. Schmiedmayer, New J. Phys. 20, 055005 (2018).

[27] M. Berry, Proc. R. Soc. A-Math. Phys. Eng. Sci. 392, 45 (1984).

[28] S.-L. Zhu and Z. Wang, Phys. Rev. Lett. 89, 097902 (2002).

[29] M. Johansson, E. Sjöqvist, L. Andersson, M. Ericsson, B. Hessmo, K. Singh, and D. Tong, Phys. Rev. A 86, 062322 (2012).

[30] S. Berger, M. Pechal, A. Abdumalikov, C. Eichler, L. Steffen, A. Fedorov, A. Wallraff, and S. Filipp, Phys. Rev. A 87, 060303 (2013).

[31] G. Feng, G. Xu, and G. Long, Phys. Rev. Lett. 110, 190501 (2013).

[32] H. Li, Y. Liu, and G. Long, Sci. China-Phys. Mech. Astron. 60, 080311 (2017).

[33] C. Zu, W. Wang, L. He, W. Zhang, C. Dai, F. Wang, and L. Duan, Nature (London) 514, 72 (2014).

[34] S. Arroyo-Camejo, A. Lazariev, S. Hell, and G. Balasubramanian, Nat. Commun. 5, 4870 (2014).

[35] C. Yale, F. Heremans, B. Zhou, A. Auer, G. Burkard, and D. Awschalom, Nat. Photonics 10, 184 (2016).

[36] Y. Sekiguchi, N. Niikura, R. Kuroiwa, H. Kano, and H. Kosaka, Nat. Photonics 11, 309 (2017).

[37] F. Kleißler, A. Lazariev, and S. Arroyo-Camejo, npj Quantum Inform. 4, 49 (2018).

[38] Y.-Y. Huang, Y.-K. Wu, F. Wang, P.-Y. Hou, W.-B. Wang, W.-G. Zhang, W.-Q. Lian, Y.-Q. Liu, H.-Y. Wang, H.-Y. Zhang, L. He, X.-Y. Chang, Y. Xu, and L.-M. Duan, Phys. Rev. Lett. 122, 010503 (2019).

[39] P. Zhao, X.-D. Cui, G. Xu, E. Sjöqvist, and D. Tong, Phys. Rev. A 96, 052316 (2017).

[40] K.-Y. Liao, X.-H. Liu, Z. Li, and Y.-X. Du, Opt. Lett. 44, 4801 (2019).

[41] B.-J. Liu, S.-L. Su, and M.-H. Yung, Phys. Rev. Research 2, 043130 (2020).

[42] L.-M. Duan, J. I. Cirac, and P. Zoller, Science 292, 1695 (2001).

[43] K. Toyoda, K. Uchida, A. Noguchi, S. Haze, and S. Urabe, Phys. Rev. A 87, 052307 (2013).

[44] D. Leibfried, B. DeMarco, V. Meyer, D. Lucas, M. Barrett, J. Britton, W. M. Itano, B. Jelenković, C. Langer, T. Rosenband, and D. J. Wineland, Nature (London) 422, 412 (2003).

[45] M.-Z. Ai, S. Li, Z. Hou, R. He, Z.-H. Qian, Z.-Y. Xue, J.-M. Cui, Y.-F. Huang, C.-F. Li, and G.-C. Guo, Phys. Rev. Applied 14, 054062 (2020).

[46] J. Zhang, L. Yan, J. Li, G. Ding, J. Bu, L. Chen, S. Su, F. Zhou, and M. Feng, Phys. Rev. Lett. 127, 030502 (2021).

[47] A. A. Abdumalikov Jr, J. M. Fink, K. Juliusson, 
M. Pechal, S. Berger, A. Wallraff, and S. Filipp, Nature (London) 496, 482 (2013).

[48] T. Yan, B.-J. Liu, K. Xu, C. Song, S. Liu, Z. Zhang, H. Deng, Z. Yan, H. Rong, K. Huang, M.-H. Yung, Y. Chen, and D. Yu, Phys. Rev. Lett. 122, 080501 (2019).

[49] Y. Xu, W. Cai, Y. Ma, X. Mu, L. Hu, T. Chen, H. Wang, Y. Song, Z.-Y. Xue, Z.-q. Yin, and L. Sun, Phys. Rev. Lett. 121, 110501 (2018).

[50] Y. Xu, Z. Hua, T. Chen, X. Pan, X. Li, J. Han, W. Cai, Y. Ma, H. Wang, Y. Song, Z.-Y. Xue, and L. Sun, Phys. Rev. Lett. 124, 230503 (2020).

[51] Z. Zhang, P. Zhao, T. Wang, L. Xiang, Z. Jia, P. Duan, D. Tong, Y. Yin, and G. Guo, New J. Phys. 21, 073024 (2019).

[52] P. Zhao, Z. Dong, Z. Zhang, G. Guo, D. Tong, and Y. Yin, Sci. China-Phys. Mech. Astron. 64, 250362 (2021).

[53] P. Boykin, T. Mor, M. Pulver, V. Roychowdhury, and F. Vatan, arXiv preprint quant-ph/9906054 (1999).

[54] M. Nielsen and I. Chuang, Quantum Computation and Quantum Information: 10th Anniversary Edition (Cambridge University Press, 2010).

[55] M. Howard, J. Twamley, C. Wittmann, T. Gaebel, F. Jelezko, and J. Wrachtrup, New J. Phys. 8, 33 (2006).
[56] K. Toyoda, K. Uchida, A. Noguchi, S. Haze, and S. Urabe, Phys. Rev. A 87, 052307 (2013).

[57] B.-J. Liu, X.-K. Song, Z.-Y. Xue, X. Wang, and M.-H. Yung, Phys. Rev. Lett. 123, 100501 (2019).

[58] Y. Song, J. Lim, and J. Ahn, Phys. Rev. Research 2, 023045 (2020).

[59] C. Parker, L.-C. Ha, and C. Chin, Nat. Phys. 9, 769 (2013).

[60] P. Hauke, M. Lewenstein, and A. Eckardt, Phys. Rev. Lett. 113, 045303 (2014).

[61] Y. Wang, A. Kumar, T.-Y. Wu, and D. Weiss, Science 352, 1562 (2016).

[62] X. Qiu, J. Zou, X. Qi, and X. Li, npj Quantum Inform. 6, 87 (2020).

[63] G. De Chiara, T. Calarco, M. Anderlini, S. Montangero, P. Lee, B. Brown, W. Phillips, and J. Porto, Phys. Rev. A 77, 052333 (2008).

[64] J. Jensen, J. Sørensen, K. Mølmer, and J. Sherson, Phys. Rev. A 100, 052314 (2019).

[65] M. Mamaev, J. H. Thywissen, and A. M. Rey, Advanced Quantum Technologies 3, 1900132 (2020).

[66] T. Hartke, B. Oreg, N. Jia, and M. Zwierlein, arXiv e-prints , arXiv:2103.13992 (2021), arXiv:2103.13992 [cond-mat.quant-gas].

[67] M. A. Nielsen and I. Chuang, "Quantum computation and quantum information," (2002). 


\title{
Supplementary Material
}

\author{
EXPERIMENTAL LATTICE SETUP
}

Our experiment is based on a Bose-Einstein condensate (BEC) of ${ }^{87} \mathrm{Rb}$ atoms confined in a harmonic trap, with trapping frequencies $\left[\left(\omega_{x}, \omega_{y}, \omega_{z}\right)=2 \pi \times(24 \mathrm{~Hz}, 48 \mathrm{~Hz}, 55 \mathrm{~Hz})\right]$ along the three spatial directions. The BEC is loaded into a one-dimensional lattice formed by counter-propagating laser beams along the $x$ direction. To achieve the holonomic quantum gate manipulation on the atom-orbital qubit, on the one hand, the lattice laser is required to be rapidly turned on and off in order to form square wave pulses in the state preparation, on the other hand, the variation of lattice depth must accurately follow the sinusoidal form in the gate operation. Our experimental setup is carefully designed with two AOMs cooperating (Fig. S1) to improve control precision. The AOM1 works with the laser power feedback control loop, consisting of PD (Photodetector), AWG1 (Arbitrary wave generator), PI (ProportionalIntegral) feedback circuit, and variable power RF (Radio frequency) source1. A voltage signal in PD for programming the lattice depth $V(t)=V_{0}+\Delta V(t)$ is achieved by the diffracted light of AOM1, where the time-dependent part $\Delta V(t)$ corresponds to a sinusoidal wave pulse. AWG1 generates the control signal $V_{A W G 1} \propto V(t)$. Both signals are connected into PI circuit, and its output controls the power of RF source1 so that the diffraction efficiency of AOM1 is delicately adjusted in order to keep the value of $V_{A W G 1}$ stable. The AOM2 works with the fast control module, consisting of RF switch, RF source2 and AWG2 that generates square wave pulses. According to RF source2, the $\mathrm{RF}$ switch can control AOM2 to realize the sharp variation of lattice depth corresponding to square wave pulses. With this feedback control design using two AOMs, the rise and fall time is maintained below 100ns, the modulation amplitude uncertainty within $2 \%$ and the laser power fluctuation below $0.2 \% @ 1$ s.

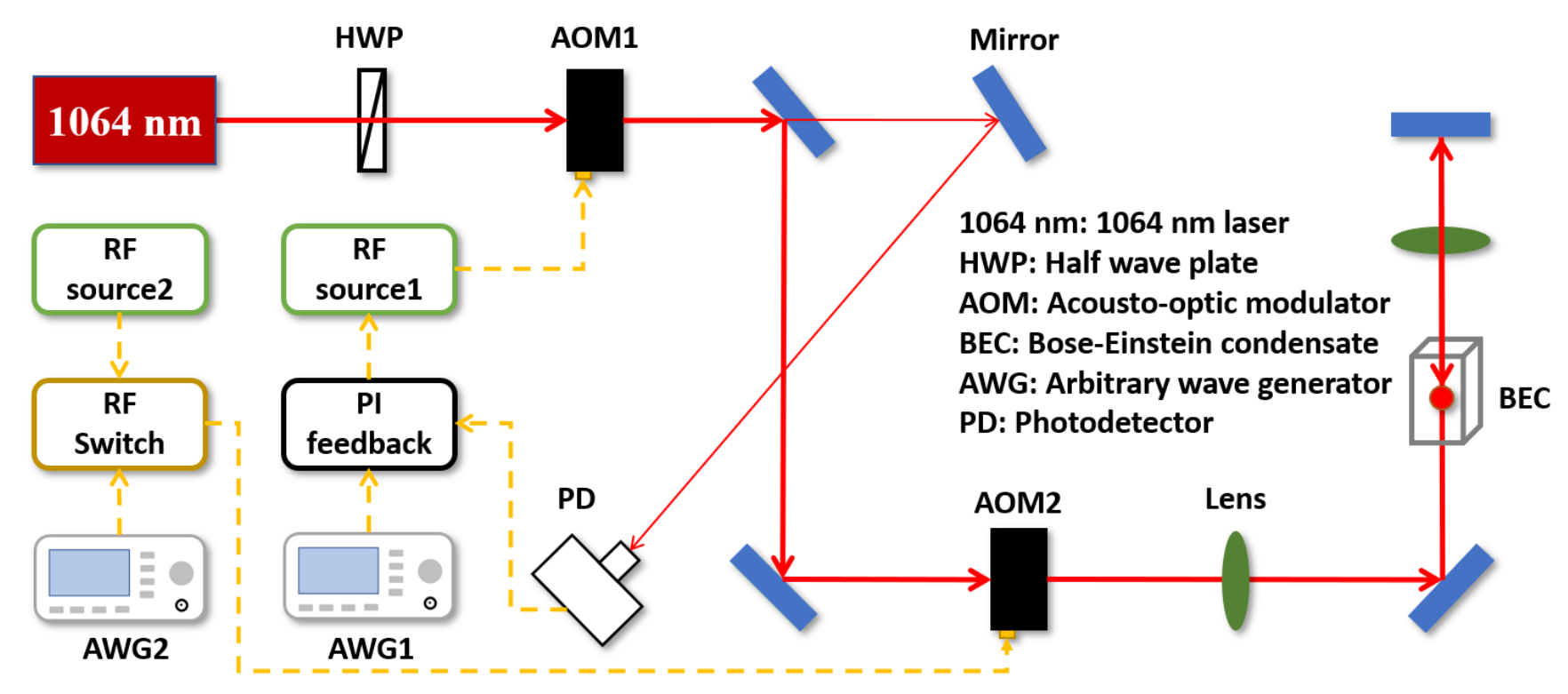

FIG. S1. Experimental set-up. The optical lattice is formed by $1064 \mathrm{~nm}$ laser, and its optical power (lattice depth) is controlled by two AOMs. The red solid line with arrow indicates the optical path and the yellow dashed line shows the direction of electrical signal transmission.

\section{QUBIT INITIALIZATION}

We use a shortcut method [26] for atom-orbital qubit initialization. After preparation of an atomic Bose-Einstein condensate in a harmonic trap, we load the atoms directly into the desired orbital state of the optical lattice. For a given targeting quantum superposition state of $|s\rangle$ and $|d\rangle$ orbitals, a pulse sequence of the lattice-forming laser is designed to maximize the state preparation fidelity (see an example in Fig. 3(a)). The shortcut method typically involves a few cycles of turn-on and off of the laser intensity, with a time duration $\tau_{\text {on }, i}$, and $\tau_{\text {off }, i}(i$ labeling different 
cycles), respectively. The time duration $\tau_{\mathrm{on}, i}$ and $\tau_{\mathrm{off}_{i}}$ defining the pulse sequence are variationally determined. The pulse sequences for preparation in the initial states $(\mathbb{S} \equiv\{|0\rangle,|1\rangle,|+\rangle,|-\rangle,|+i\rangle,|-i\rangle\})$ are provided in Table $\mathrm{S} 1$. This shortcut method is demonstrated an efficient protocol for atom-orbital qubit initialization.

\begin{tabular}{ccccccc}
\hline Initial state & $\tau_{\text {on }, 1}$ & $\tau_{\text {off }, 1}$ & $\tau_{\text {on }, 2}$ & $\tau_{\text {off }, 2}$ & $\tau_{\text {on }, 3}$ & $\tau_{\text {off }, 3}(\mu s)$ \\
\hline$|0\rangle$ & 64.5 & 40.7 & 62.1 & 41.4 & & \\
$|1\rangle$ & 28.1 & 35.1 & 40.6 & 27.0 & & \\
$|+\rangle$ & 31.0 & 7.7 & 1.5 & 41.1 & 12.1 & 8.7 \\
$|-\rangle$ & 38.4 & 61.7 & 38.6 & 30.7 & & \\
$|+i\rangle$ & 52.0 & 80.0 & 3.0 & 4.0 & & \\
$|-i\rangle$ & 23.3 & 43.7 & 56.5 & 41.8 & & \\
\hline
\end{tabular}

TABLE S1. The laser pulse sequences for atom-orbital qubit initialization.

\section{PULSE SEQUENCE DESIGN}

In constructing the atom-orbital qubit and the holonomic quantum control, we have neglected atomic interactions, which is valid in a relatively shallower lattice. The requirement of having unharmonic band structures to avoid unwanted inter-band transitions also favors shallow lattice. At the same time, the lattice cannot be too shallow for the corresponding coupling $\lambda$ would be too small to maintain the validity of the rotating wave approximation as used in constructing the holonomic dynamical invariant (Eq. (4)). Taking these constraints into account, we choose a moderate lattice depth of $V_{0}=5 E_{r}$. We have also tried other lattice depths in the experiment, and the choice of $5 E_{r}$ is found to perform the best.

Through numerical tests, we observe that a single holonomic unitary operation given Eq. (6) is not adequate to produce universal SU(2) quantum gates. To resolve this problem, we consider a concatenated unitary composed of a sequence of holonomic unitary operations,

$$
U=U_{\beta_{M} \varphi_{M}} U_{\beta_{M-1} \varphi_{M-1}} \ldots U_{\beta_{2} \varphi_{2}} U_{\beta_{1} \varphi_{1}}
$$

in order to construct generic quantum gates. The control sequences $\left(\beta_{j}, \varphi_{j}\right)$ are obtained by optimizing the gate fidelity,

$$
F=\mid \operatorname{tr}\left(U^{\dagger} U \text { target }\right) \mid / 2 \text {, }
$$

with $U_{\text {target }}$ the targeting gate. Having $\left(\beta_{j}, \varphi_{j}\right)$, the phase of the laser pulse in Eq.(2) is given, and the modulation amplitude is given by

$$
A=\frac{|\Delta \tan \beta|}{\int d x V_{p}(x) \phi_{d}(x) \phi_{s}(x)} .
$$

Considering the physical setup, we have $A \in[0,1]$, this sets a constraint on $\beta, \beta \in[0,0.2]$. In absence of the phase $\varphi$ controlability, the lattice setup would be too restrictive to produce a proper holonomic control unitary with a satisfactory fidelity (Eq. (S2)), which is the reason for us to introduce the control over the phase $\varphi$ in the experiment. Having both $\beta$ and $\varphi$ under control, we find it is adequate to construct the holonomic $X, Y, Z$, Hadamard, and $\pi / 8$ gates with $M \leq 5$. The corresponding control sequences are listed in Table. S2.

\section{TIME-OF-FLIGHT QUANTUM STATE TOMOGRAPHY}

In our experiment, the momentum distribution $n(\tilde{p})$ (with $\tilde{p}$ the momentum along the lattice direction) is obtained with a standard tight-of-flight measurement. For the lattice BEC with orbital degrees of freedom, the momentum distribution is related to the density matrix in Eq. (1) by,

$$
n(\tilde{p}) \propto \sum_{\ell, \nu, \nu^{\prime}} \delta_{\tilde{p} l, 4 \ell \pi} \rho_{\nu \nu^{\prime}} \tilde{w}_{\nu}(\tilde{p}) \tilde{w}_{\nu^{\prime}}^{*}(\tilde{p}),
$$

with $\tilde{w}_{\nu}(\tilde{p})$ the Fourier transform of of the Wannier function of the $\nu$-orbital band. The momentum distribution has coherent peaks at $\tilde{p}=4 \ell \pi / l$. In principle, the density matrix can be fully reconstructed from the measured momentum 
TABLE S2. Sequences of the holonomic quantum gates before the orbital leakage elimination. Each pulse is applied to the system for one entire period of time $(2 \pi / \omega)$.

\begin{tabular}{|c|c|c|c|c|c|}
\hline gate & & pulse 1 pulse 2 & pulse 3 & pulse 4 & ulse 5 \\
\hline \multirow{3}{*}{$\mathrm{X}$-gate } & $\omega /(2 \pi \cdot \mathrm{kHz})$ & $\begin{array}{ll}10.768 & 10.777\end{array}$ & 10.769 & 10.772 & \\
\hline & $A$ & $0.6332 \quad 0.6505$ & 0.6351 & 0.6417 & \\
\hline & $\varphi / \mathrm{rad}$ & $1.5377 \quad 1.7139$ & 1.5816 & 1.2258 & \\
\hline \multirow{3}{*}{ Y-gate } & $\omega /(2 \pi \cdot \mathrm{kHz})$ & 10.63410 .736 & 10.892 & 10.998 & \\
\hline & $A$ & $0.2949 \quad 0.5708$ & 0.8311 & 0.9697 & \\
\hline & $\varphi / \mathrm{rad}$ & $1.0246 \quad 0.0415$ & 0.0118 & 0.0141 & \\
\hline \multirow{3}{*}{ Z-gate } & $\omega /(2 \pi \cdot \mathrm{kHz})$ & 10.89210 .886 & 10.864 & 10.889 & 10.896 \\
\hline & $A$ & $0.8318 \quad 0.8231$ & 0.7913 & 0.8274 & 0.8375 \\
\hline & $\varphi / \mathrm{rad}$ & $0.5454 \quad 0.7938$ & 1.5684 & 2.3366 & 2.5971 \\
\hline \multirow{3}{*}{ Hadamard } & $\omega /(2 \pi \cdot \mathrm{kHz})$ & $10.815 \quad 10.783$ & 10.770 & 10.796 & 10.836 \\
\hline & $A$ & $0.7147 \quad 0.6611$ & 0.6375 & 0.6828 & 0.7493 \\
\hline & $\varphi / \mathrm{rad}$ & $2.3780 \quad 2.1068$ & 1.5938 & 1.0489 & 0.6591 \\
\hline \multirow{3}{*}{$\pi / 8$-gate } & $\omega /(2 \pi \cdot \mathrm{kHz})$ & $11.023 \quad 11.023$ & 11.023 & 11.023 & 11.023 \\
\hline & $A$ & $0.9987 \quad 0.9987$ & 0.9987 & 0.9987 & 0.9987 \\
\hline & $\varphi / \mathrm{rad}$ & $1.4075 \quad 1.4860$ & 1.5645 & 1.6431 & 1.7216 \\
\hline
\end{tabular}

distribution. But in practice, the higher order momentum peaks with $|\ell| \geq 2$ have rather low signal-to-noise ratio compared to $\ell=0, \pm 1$. Having the momentum distribution at $\ell=0, \pm 1$ only is not adequate to map out the density matrix.

For quantum state tomography, we let the state to measured evolve in the static lattice for a certain amount of time $t_{\text {evo }}$. The time dependence of the momentum distribution $n\left(\tilde{p} ; t_{\text {evo }}\right)$ is given by Eq. (S4) with $\rho_{s d}\left(\rho_{d s}\right)$ replaced by $\rho_{s d} e^{-i \Delta \times t_{\mathrm{evo}} / \hbar}\left(\rho_{d s} e^{i \Delta \times t_{\mathrm{evo}} / \hbar}\right)$. With experimental data for $n\left(\tilde{p} ; t_{\mathrm{evo}}\right)$, the full density matrix $\rho_{\nu \nu^{\prime}}$ is mapped out by fitting. This quantum state tomography scheme for orbital states is analogous to a quench protocol developed for tomography of band insulator topology [60].

\section{CONSTRUCTION OF RANDOM UNITARY GATES}

To justify the universality of holonomic gate construction for all single-qubit gates, we randomly sample the $\mathrm{SU}(2)$ unitary gates, and obtain its fidelity in atom-orbital gate realization through our multi-orbital simulation method, which has been found to model the experiment quantitatively. For an $\mathrm{SU}(2)$ unitary operation, parameterized as $e^{i \sigma(\theta, \phi) \beta / 2}$, with $\beta$ the rotation angle on the Bloch sphere, and $\theta$ and $\phi$ defining the rotation axis $-\sigma(\theta, \phi)=\cos (\theta) \sigma_{z}+$ $\sin (\theta)\left[\cos (\phi) \sigma_{x}+\sin (\phi) \sigma_{y}\right]$. We randomly sample the angles according to a uniform distribution, $\theta \in[0, \pi), \phi \in$ $[0,2 \pi)$, and $\beta \in[0,2 \pi)$. Through the orbital leakage error elimination protocol, we confirm all these generated random unitary gates can be performed with our atom-orbital qubit, with final state fidelities all above 98\% (Figure S2).

\section{ROBUSTNESS AGAINST LASER FLUCTUATIONS}

Having robustness against noise in the quantum control protocol is critical to reaching high quality quantum information processing. We characterize the robustness of the holonomic quantum gates by deliberately introducing a slight deviation on the optical lattice potential amplitude in Eq. (2) with an amount of $\delta A$. The results of the robustness study are provided in Fig. S3. As the lattice modulation amplitude varies up to 10\%, we find the average experimental fidelity of the holonomic gates remains above $90 \%$. The experimental results have a quantitative agreement with the multi-orbital numerical simulation (Fig. S3(a)).

In order to further illustrate the advantage of the holonomic gates, we also construct a non-holonomic dynamic gate, and show their comparison in Fig. S3(a). As Fig. S3(b) shows, the non-holonomic dynamic gate is constructed by a series of square wave pulses for the lattice depth, and its dynamic phase is not cancelled. The time duration and interval in each pulse are optimized to reach the targeting gate operation. The specific time sequences are shown in Fig. S3(c), and the total time is close to the holonomic gate for a fair comparison. Both gates can achieve high fidelity without the laser intensity noise (when $\delta A / A=0$ ). However, by introducing laser intensity noise with $\delta A / A \neq 0$, we find that the holonomic gates exhibit better resistance against fluctuations. For example, the QPT fidelity of the holonomic $\mathrm{X}$-gate is above $92 \%$ when the fluctuation $\delta A / A$ reaches $20 \%$. In sharp contrast, the fidelity of the 


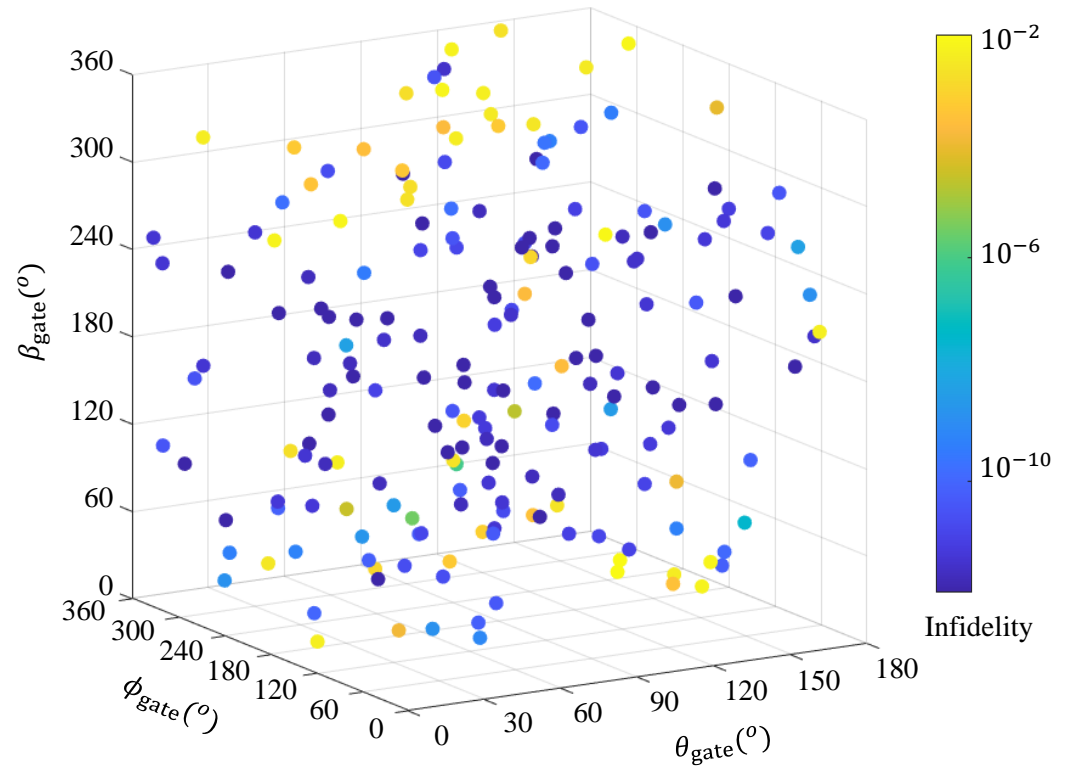

FIG. S2. Final state fidelities of 100 random SU(2) rotations. We randomly sample rotation axis (represented by the polar angle $\theta_{\text {gate }}$ and the azimuth angle $\phi_{\text {gate }}$ ) and the rotation angle $\beta_{\text {gate }}$. For each of these quantum gates, we theoretically design the holonomic quantum control sequence and calculate the corresponding final state fidelity using our multi-orbital numerical simulations. All the gates are achieved with the pulse step $M \leq 5$. All fidelities are above $98 \%$.

(a1)

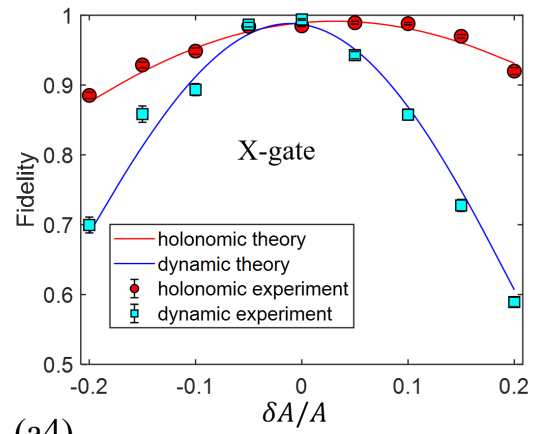

(a4)

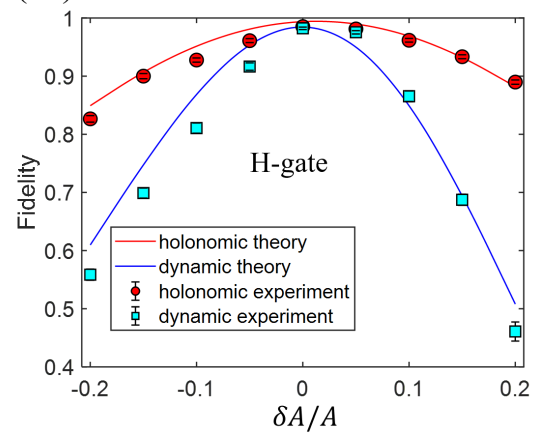

(a2)

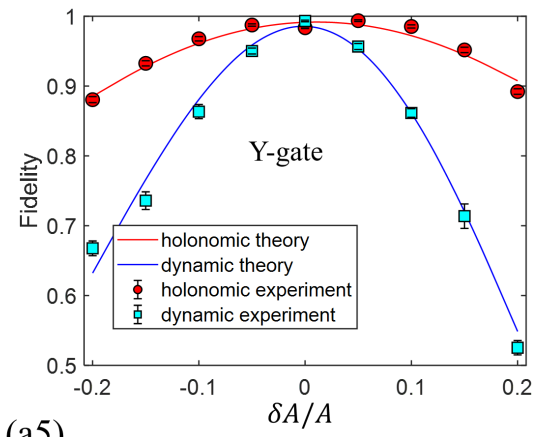

(a5)

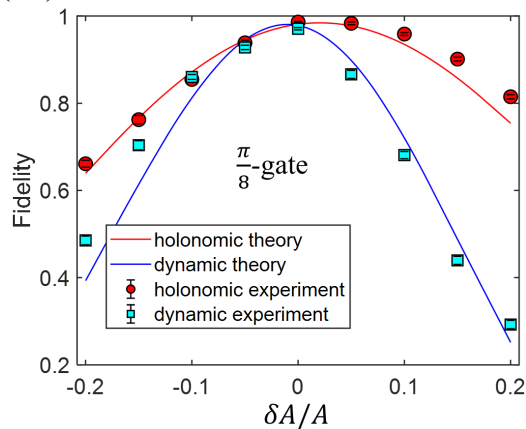

(a3)

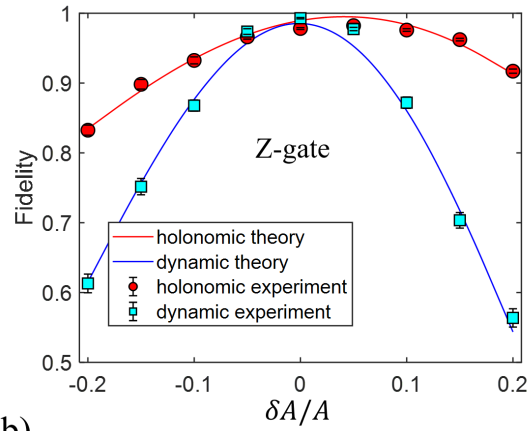

(b)

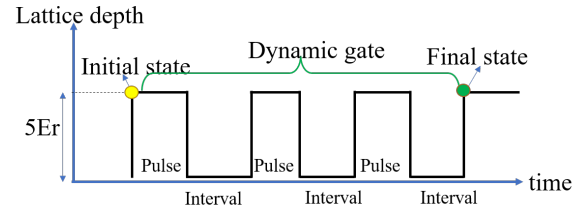

(c)

\begin{tabular}{|c|c|c|c|c|c|c|}
\hline Dynamic/ $\mu \mathrm{s}$ & Pulse & Interval & Pulse & Interval & Pulse & Interval \\
\hline X-gate & 188.7 & 35.3 & 74.3 & 29.1 & 74.3 & 35.3 \\
\hline Z-gate & 144.3 & 36.1 & 97.7 & 42.2 & 97.7 & 36.1 \\
\hline Y-gate & 142.9 & 36.9 & 164.6 & 30.0 & 77.7 & 33.4 \\
\hline H-gate & 141.6 & 15.3 & 87.5 & 61.7 & 132.0 & 3.30 \\
\hline pi/8-gate & 132.9 & 12.3 & 177.5 & 21.6 & 124.1 & 36.5 \\
\hline
\end{tabular}

FIG. S3. Comparison between holonomic gates and non-holonomic dynamic gates. (a) Gate robustness against laser fluctuation noise. The ratio $(\delta A / A)$ represents the relative fluctuation strength in the laser intensity. DC-noise is applied here to account for long-term drift of laser intensities that may potentially happens in experiments. The red points (blue squares) represent the experimental fidelity of the holonomic gates (dynamic gates). The fidelity is obtained by the QPT method. The red lines (blue lines) are the theoretical results (simulated with the multi-orbital numerical method) for the fidelity of the holonomic gates (dynamic gates). (a1) to (a5) correspond to the X-, Y-, Z-, Hadamard-, and $\pi / 8$ - gate, respectively. (b) Time sequence of the non-holonomic dynamic gate. The dynamic gate is constructed by a series of square wave pulses. The duration and interval for each pulse are designed and optimized to realize different gates. The pulse sequence details are provided in (c). 


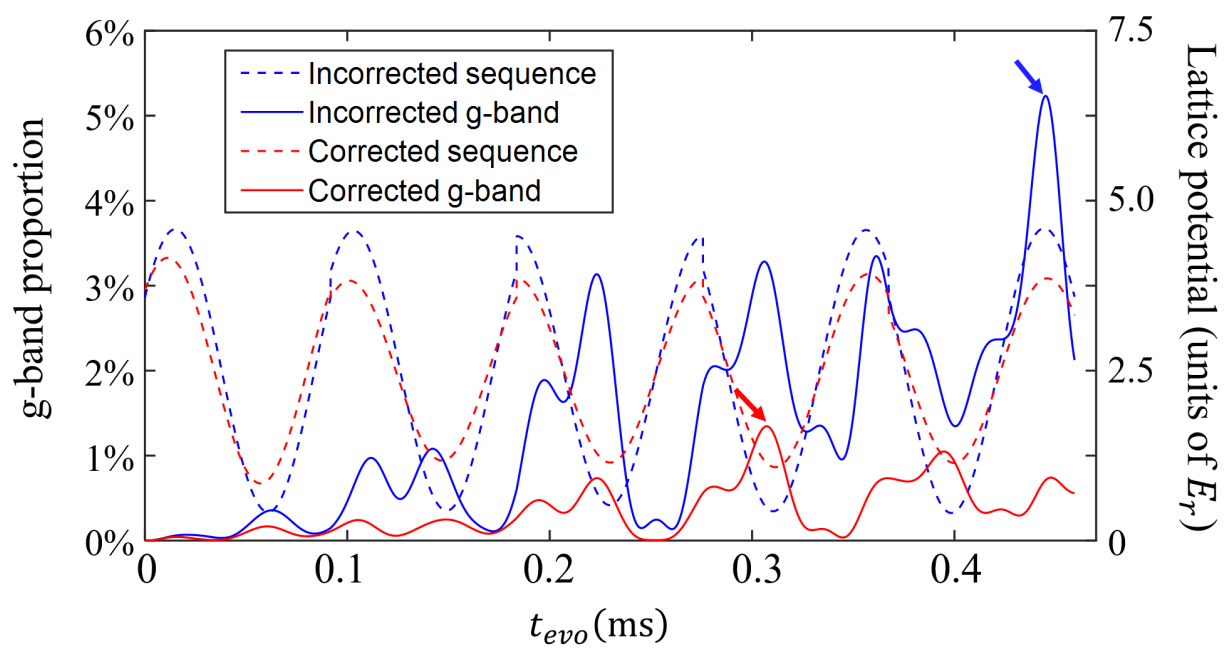

FIG. S4. Leakage to the $g$-band. The red dashed and blue dashed lines represent the modulation sequences of the $\mathrm{Z}$ gate with and without our orbital leakage elimination scheme, respectively. The red solid and blue solid lines illustrate the proportion of leakage to the $\mathrm{g}$-band during the $\mathrm{Z}$ gate process with and without the orbital leakage elimination. The red and blue arrows mark the maximum proportion of the $g$-band for the two cases. Here we choose the initial state to be $|1\rangle$.

non-holonomic dynamic X-gate is below $60 \%$. The noise resilience of the holonomic quantum gates on orbital qubits is thus confirmed in our experiment.

In principle, imperfect control of the pulse durations may also affect the gate quality. But in optical lattice experiments, the control precision of pulse duration is typically maintained below the level of $10^{-4}$, which could only cause fidelity decreasing by about $0.01 \%$ through our numerical simulation.

\section{ORBITAL LEAKAGE ELIMINATION}

In the experiment, we observe that the orbital leakage error strongly compromises the orbital qubit gate fidelity. Although we try to avoid the transitions to the unwanted orbitals by energy suppression and symmetry, the leakage error is still present and harmful. As an example, we show the process of Z-gate operation on the initial state $|1\rangle$ in Fig. S4. Through our multi-orbital numerical simulation, we find that $g$-band proportion reaches above $5 \%$, and the final state fidelity is about $71 \%$.

In order to resolve the orbital leakage problem, we develop an orbital leakage elimination technique, to be described in the next paragraph. With the orbital leakage elimination technique, the proportion of the g-band drops down below $1.3 \%$ (Fig. S4), and the fidelity reaches above $99 \%$ for the Z-gate operation, in our numerical simulation. The orbital leakage elimination technique is implemented in our experiment. This technique is the key for us to reach the high gate fidelities as reported in the main text.

Here we describe the details of orbital leakage elimination technique. For a target holonomic quantum gate, we first obtain the parameter sequence $\left(\theta_{j}, \varphi_{j}\right)$ by maximizing the idealized gate fidelity (the index $j$ labels the lattice modulation periods). Then at each lattice modulation period, the quantum state evolution $U^{\star}(t)(t \in[0, T])$ is fully specified by $\theta_{j}$ and $\varphi_{j}$. The orbital leakage elimination scheme is to search for a most suitable choice of lattice modulation pulse, i.e., $(\omega, A, \varphi)$, which gives a quantum evolution that best approximates $U^{\star}(t)$. In order to reach this solution, we propose a loss function

$$
\begin{aligned}
L(\Theta)= & \int d t\left\{\Sigma_{n m \in\{S, D\}}\left|U_{n m}^{\star}(t)-U_{n m}(t ; \Theta)\right|^{2}+\right. \\
& \left.\Sigma_{n \in\{S, D\}, m \notin\{S, D\}}\left|U_{n m}(t ; \Theta)\right|^{2}+\left|U_{m n}(t ; \Theta)\right|^{2}\right\},
\end{aligned}
$$

where $\Theta$ represents $(\omega, A, \varphi)$, and $U(t ; \Theta)$ the quantum evolution matrix incorporating all orbitals in our calculation. Through minimization, we obtain a most suitable $\Theta$, with which the leakage error caused by the unwanted orbitals is minimized. The control sequences designed with the orbital leakage error elimination protocol for $X, Y, Z$, Hadamard, and $\pi / 8$ gates are listed below as Table. S3. 


\begin{tabular}{|c|c|c|c|c|c|c|}
\hline gate & & & & & & \\
\hline \multirow{3}{*}{$\mathrm{X}$-gate } & $\omega /(2 \pi \cdot \mathrm{kHz})$ & 10.768 & 10.777 & 10.769 & 10.772 & \\
\hline & $A$ & 0.4749 & 0.4555 & 0.5626 & 0.4765 & \\
\hline & $\varphi / 1$ & 1.6142 & 1.6383 & 1.5791 & 1.2129 & \\
\hline \multirow{3}{*}{ Y-gate } & $\omega /(2 \pi \cdot \mathrm{kHz})$ & 10.634 & 10.736 & 10.892 & 10.998 & \\
\hline & $A$ & 0.2027 & 0.3952 & 0.6141 & 0.7747 & \\
\hline & $\varphi / \mathrm{rad}$ & 0.6247 & 0.2082 & 0.1576 & 0.1429 & \\
\hline \multirow{3}{*}{ Z-gat } & $\omega /(2 \pi \cdot \mathrm{kHz})$ & 10.892 & 10 . & 10.864 & 10.889 & \\
\hline & A & 0.6639 & 0.5 & 0. & 80 & \\
\hline & $\varphi / \mathrm{rad}$ & 0.7 & 0.9442 & 1.5551 & 2.2610 & \\
\hline \multirow{3}{*}{ Hadamard } & $\omega /(2 \pi \cdot \mathrm{kHz})$ & 10.815 & 10.783 & 10.770 & 10.796 & 10 \\
\hline & $A$ & 0.5153 & 0.5 & 0.5445 & 0.5281 & \\
\hline & $\varphi / \mathrm{rad}$ & 2.3395 & 2.0068 & 1.5531 & 1.1157 & 0.8583 \\
\hline \multirow{3}{*}{$\pi / 8$-gate } & $\omega /(2 \pi \cdot \mathrm{kHz})$ & 11.023 & 11.023 & 11.023 & 11.023 & 11.023 \\
\hline & $A$ & 0.8012 & 0.8030 & 0.7970 & 0.8176 & 0.6218 \\
\hline & $\varphi / \mathrm{rad}$ & 1.5058 & 1.4766 & 1.5636 & 1.6471 & 1.6892 \\
\hline
\end{tabular}

TABLE S3. The control sequences of the holonomic quantum gates with the orbital leakage elimination protocol. Each pulse is applied to the system for one entire period of time $(2 \pi / \omega)$.

\section{MULTI-ORBITAL SIMULATION METHOD}

In the experiment, we find the discrepancy in the simplified two-orbital model in Eq. (4) - the simulated results have significant disagreement with the experiment. We thus develop a multi-orbital simulation method, which treats the non-interacting quantum dynamics exactly. Having atoms confined in a dynamical potential $V(x, t)=V(t) \cos ^{2}(K x)$, the Bose-Einstein condensate state evolves in a subspace spanned by plane wave states $|q\rangle$, with its wave function $e^{2 i q K x}$. In this subspace, the kinetic part of the Hamiltonian is diagonal given by

$$
H_{K, q q^{\prime}}=\frac{\hbar^{2}}{2 M}(2 q K)^{2} \delta_{q q^{\prime}}
$$

The potential term is off-diagonal given by

$$
H_{P, q q^{\prime}}=\frac{1}{2} V(t) \times\left(\delta_{q, q^{\prime}+1}+\delta_{q, q^{\prime}-1}\right) .
$$

The quantum state evolution is described by a time-dependent column vector $\psi(t)$, according to

$$
\psi(t+\delta t)=e^{-i H_{K} \delta t / 2} e^{-i H_{P}(t+\delta t / 2) \delta t} e^{-i H_{K} \delta t / 2} \psi(t)+\mathcal{O}\left(\delta t^{3}\right) .
$$

Since $H_{K}$ is diagonal, the multiplication of $e^{-i H_{K} \delta t}$ on a vector can be efficiently implemented as multiplication of an element-wise phase. The matrix $H_{P}$ is off-diagonal, but can be diagonalized by a Fourier transformation. The matrix multiplication of $e^{-i H_{K} \delta t}$ can be efficiently performed by combining with a Fast Fourier transformation algorithm. Choosing a high-momentum cutoff $|q|<q_{\max }$, the computation complexity of this multi-orbital simulation method is $\mathcal{O}\left(q_{\max } \log q_{\max }\right)$. The simulated results have very good agreement with our experimental measurements (Fig. S5). With numerical simulation, we find the orbital leakage error strongly damages the quantum gate operations. With our orbital leakage elimination protocol, the time evolution taking the corrected control pulses approaches to the ideal holonomic quantum dynamics.

\section{CONSTRUCTION OF MULTIPLE QUBITS AND TWO-QUBIT OPERATIONS}

In our experiment, we have performed single qubit control based on the orbital state of a BEC. To engineer scalable quantum circuits with the orbital qubit setup, we need to introduce single-site controllablility, which is experimentally accessible with quantum microscope techniques [61,62]. To selectively control the orbital transition on one targeting site, say at $x_{0}$, we propose to maintain the optical potential approximately homogeneous elsewhere, and tune the gap between $s$ and $d$ orbitals at site $x_{0}$ far off-resonant from other sites by manipulating the local potential using quantum microscope techniques. The overall optical potential is now denoted as $\tilde{V}(x)$, and the gap between $s$ and $d$ orbitals at the targeting site is $\tilde{\Delta}_{0}$. The orbital transition at $x_{0}$ can then be controlled following the same way as demonstrated experimentally in this work, namely by introducing a modulating lattice potential,

$$
\Delta \tilde{V}(x, t)=A \sin (\omega t+\varphi) \tilde{V}(x)
$$



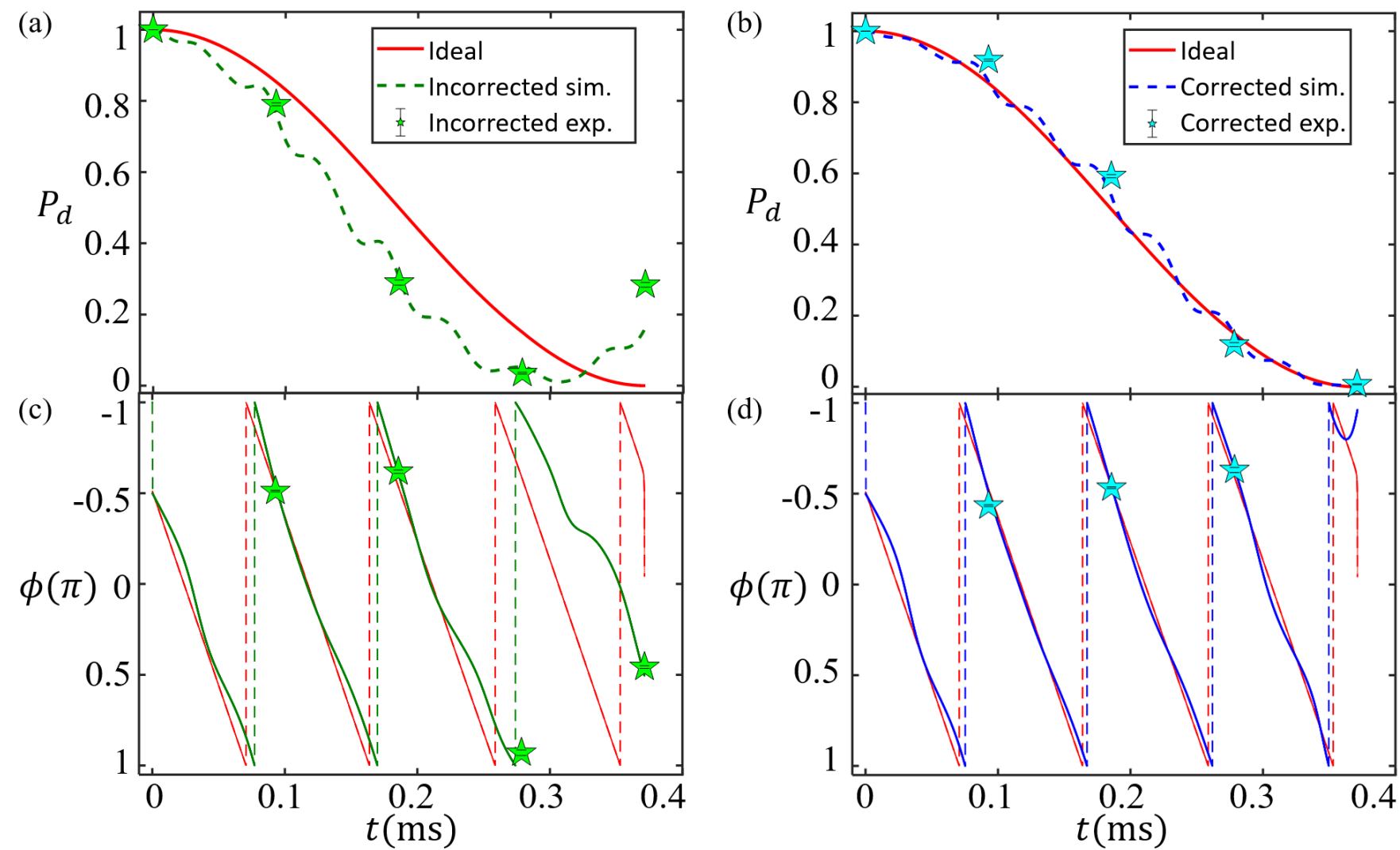

FIG. S5. Comparison between experimental observation with numerical simulation for the quantum state evolution during the holonomic quantum control. The dynamical process corresponds to a holonomic $X$-gate on the initial state, $|0\rangle(d$-orbital state). (a) and (b) show the time evolution of the proportion of the $d$-band population, $P_{d}$. (c) and (d) show the time evolution of the phase difference between $|0\rangle$ ( $d$-orbital state) and $|1\rangle$ (s-orbital state). The red lines represent the ideal state evolution of the holonomic control. The blue and green stars correspond to the experimental results with and without the orbital leakage elimination protocol. The green and blue lines are the corresponding simulation results.

The modulation frequency $\omega$ should be chosen to match the gap $\tilde{\Delta}_{0}$. Working in the limit of one particle per lattice site, the interaction effects during the control process of the single-qubit rotation are negligible. The integration of quantum microscope techniques to the present holonomic orbital qubit control would make an orbital-based quantum computing platform with single-qubit addressibility.

The two-qubit gate can be achieved by adapting the atom collision scheme [63-66]. Considering two nearby sites, denoted as $a$ and $b$, the orbital states are $\left|s_{a} s_{b}\right\rangle,\left|d_{a} d_{b}\right\rangle,\left|s_{a} d_{b}\right\rangle$, and $\left|d_{a} s_{b}\right\rangle$. The local interactions projected to this subspace give a vanishing contribution for each site only contains one particle. The leading order interaction that contributes to the dynamics in the two-qubit computation subspace is thus between neighboring sites. Given the presence of a large gap between $s$ and $d$ orbitals, the interaction processes that do not respect the conservation of single-particle energy are negligible. The relevant interactions between neighboring sites are then

$$
H_{\mathrm{int}, \mathrm{nn}}=U_{1} \hat{s}_{a}^{\dagger} \hat{s}_{b}^{\dagger} \hat{s}_{b} \hat{s}_{a}+U_{2} \hat{d}_{a}^{\dagger} \hat{d}_{b}^{\dagger} \hat{d}_{b} \hat{d}_{a}+U_{3}\left(\hat{s}_{a}^{\dagger} \hat{d}_{b}^{\dagger} \hat{d}_{b} \hat{s}_{a}+\hat{d}_{a}^{\dagger} \hat{s}_{b}^{\dagger} \hat{s}_{b} \hat{d}_{a}\right)+U_{4}\left(\hat{s}_{a}^{\dagger} \hat{d}_{b}^{\dagger} \hat{s}_{b} \hat{d}_{a}+\hat{d}_{a}^{\dagger} \hat{s}_{b}^{\dagger} \hat{d}_{b} \hat{s}_{a}\right)
$$

where we have introduced orbital annihilation operators $\hat{s}_{a}, \hat{s}_{b}, \hat{d}_{a}, \hat{d}_{b}$ on the two sites. For the orthogonality between different wannier functions on each site, $U_{4}$ is much smaller than $U_{3}$, and can be neglected. The interactions $U_{1}$ and $U_{2}$ only contribute to the diagonal terms in the two-qubit computation subspace. The $U_{3}$ process is more nontrivial, causing a swap operation between $\left|s_{a} d_{b}\right\rangle$ and $\left|d_{a} s_{b}\right\rangle$. The swap operation is completed with the interaction turned on for a time duration

$$
\tau_{\text {swap }}=\pi \hbar /\left(2 U_{3}\right)
$$

A $\sqrt{\text { swap }}$ gate is then reached by letting atoms interact for a time duration

$$
\tau_{\sqrt{\text { swap }}}=\tau_{\text {swap }} / 2 \text {. }
$$


This makes an entangling two-qubit gate, which is sufficient to make a universal gate set by combining with single-

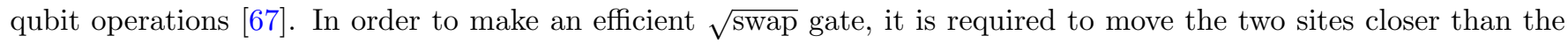
lattice spacing by adiabatic time evolution. This requires quantum microscope or sublattice techniques. 